\title{
Prognostic impact of hepatitis B or C on intrahepatic cholangiocarcinoma
}

Jung Woong Seo*, Byung Soo Kwan ${ }^{*}$ Young Koog Cheon, Tae Yoon Lee, Chan Sup Shim, So Young Kwon, Won Hyeok Choe, Byung Chul Yoo, Jeong Min Yoon, and Jung Hoon Lee

Division of Gastroenterology, Department of Internal Medicine, Konkuk University School of Medicine, Seoul, Korea

Received: February 12, 2018

Revised : June 12, 2019

Accepted: July 11, 2019

\section{Correspondence to}

Young Koog Cheon, M.D.

Division of Gastroenterology,

Department of Internal

Medicine, Konkuk University

School of Medicine, 120

Neungdong-ro, Gwangjin-gu,

Seoul 05029, Korea

Tel: +82-2-2030-7490

Fax: +82-2-2030-7458

E-mail: ykskyoo1@hanmail.net

*These authors contributed equally to this work.
Background/Aims: Intrahepatic cholangiocarcinoma (ICC) is the second-most common primary liver malignancy, arising from the peripheral intrahepatic bile duct epithelium. Hepatitis B virus (HBV) or hepatitis C virus (HCV) may be involved in the development of ICC. We explored the prognostic value of hepatitis virus infection, as well as other prognostic factors affecting survival in patients with ICC.

Methods: A retrospective chart review was performed for patients diagnosed with ICC between August 2005 and December 2018 at Konkuk University Medical Center. We identified a total of 131 patients with ICC. Overall survival rates of patients with and without hepatitis were determined. Univariate and multivariate analyses were used to estimate factors influencing survival outcomes.

Results: A total of $17.6 \%$ (23/131) of patients were positive for HBV or HCV. Hepatitis B positive ICC patients were significantly younger with higher albumin and higher $\alpha$-fetoprotein than those without hepatitis viral infections. The median survival of hepatitis-positive and hepatitis-negative groups was 280 and 213 days, respectively. Survival rates were not significantly different between the two groups $(p=0.279)$. Multivariate analyses indicated that lower serum carbohydrate antigen 19-9 (CA 19-9) $(p<0.001)$, lower T stage $(p=0.042)$, the absence of lymph-node metastasis $(p=0.043)$, and receiving curative surgery $(p=0.033)$ were independent predictors of better outcomes.

Conclusions: While hepatitis influenced a number of clinical features in ICC patients, it did not affect survival rate. Prognostic factors influencing survival outcomes with ICC were CA 19-9 level, T stage, the presence of lymph node metastasis, and curative surgery.

Keywords: Intrahepatic cholangiocarcinoma; Hepatitis B virus; Hepatitis C virus; Survival rate

\section{INTRODUCTION}

Intrahepatic cholangiocarcinoma(ICC), the second-most common primary hepatic tumor (after hepatocellular carcinoma [HCC]), accounts for approximately $3 \%$ of all gastrointestinal tumors and 10\% to $15 \%$ of primary liv- er malignancies $[1,2]$. The incidence and mortality rates of ICC have been increasing globally in recent decades. ICC is a lethal malignancy, with a median survival of 6.5 months from the time of diagnosis in untreated patients [3]. However, the etiology of cholangiocarcinoma remains unclear. The primary risk factor for ICC is pri- 
mary sclerosing cholangitis. Obesity, inflammatory conditions of the biliary tract, ulcerative colitis, smoking, Caroli's disease, exposure to radiopaque medium thorium dioxide (Thorotrast, Testagar \& Co. Inc., Detroit, MI, USA), and diabetes have also been proposed as risk factors $[1,3]$. Hepatitis viruses, including hepatitis B virus (HBV) and hepatitis $\mathrm{C}$ virus (HCV), are causative agents of HCC. Recent studies have shown that both HBV and HCV infections can cause ICC $[4,5]$, which may explain its increasing incidence. Regardless of etiology, surgical resection offers the only prospect of cure; however, only a small percentage of patients with ICC have resectable cancer at the time of diagnosis.

Given the increasing incidence of ICC, its poor prognosis, and the lack of adequate treatment options, further studies exploring its risk factors, prognostic indicators, and outcomes are required. While several recent studies have indicated that HBV and HCV infections have prognostic significance for ICC [6,7], the prognostic impact of such infections remains controversial. Therefore, we performed a retrospective study of all patients with ICC treated between 2005 and 2018 at a single institution to evaluate the prognostic value of hepatitis virus infection status and to identify other prognostic factors that affect survival in patients with ICC.

\section{METHODS}

\section{Patients}

A retrospective chart review was performed for patients diagnosed with ICC between August 2005 and December 2018 at Konkuk University Medical Center. All patients with an intrahepatic mass were evaluated for operability by computed tomography (CT), magnetic resonance imaging (MRI), endoscopic retrograde cholangiopancreatography, or magnetic resonance cholangiopancreatography. Ultrasound-guided biopsies were performed to confirm the pathologic diagnosis when patients were assessed with unresectable ICC by radiologic examination. Patients with HCC-cholangiocarcinoma mixed type, intraductal papillary biliary neoplasm, periductal infiltrative cholangiocarcinoma, or intraductal growing type were excluded. Only the mass-forming type was included. Patients with extrahepatic or hilar cholangiocarcinoma, neuroendocrine tumor, or sarcoma were excluded.
Serologic tests were performed for hepatitis B surface antigen (HBsAg), anti-HCV, indicators of liver function, carbohydrate antigen 19-9 (CA 19-9), and $\alpha$-fetoprotein (AFP) level. Tumor-node-metastasis (TNM) staging of ICC was performed according to the 7 th edition of the American Joint Committee on Cancer (AJCC) staging systems. Surgical procedures were divided into major and minor resections. Major resection included hemihepatectomy and central bisectionectomy, while minor resection included sectionectomy and segmentectomy. This study was approved by the Institutional Review Board of Konkuk University Medical Center (KUH 1010778). Written informed consent by the patients was waived due to a retrospective nature of our study.

\section{Statistical analysis}

Statistical analyses were performed using SPSS software version 18.o (SPSS Inc., Chicago, IL, USA). Statistical significance was defined as $p<0.05$. Survival of patients in the study group was measured from the time of diagnosis. The study groups were compared using the chisquare test or Fisher's exact test for categorical variables and Wilcoxon's rank-sum test for continuous variables. Overall survival with HBV infection status was calculated using the Kaplan-Meier method. Log-rank tests were used to assess differences. Univariate and multivariate analyses were performed using the Cox proportional-hazards model, with the backward Wald procedure.

\section{RESULTS}

Of the 635 patients diagnosed with cholangiocarcinoma during the study period, 131 patients had ICC (Fig. 1). Table 1 describes the clinical features of the patients in the study group. It included 74 males and 57 females, with a mean age of $66.6 \pm 11.7$ years. Among these, a total of 17.6\% (23/131) were positive for either HBsAg (19/23) or anti-HCV (4/23). The distribution among the seven different AJCC stages was as follows: I (14.5\%), II (9.9\%), III (2.3\%), IVa (34.4\%), and IVb (38.9\%). Of the 131 patients, 37 underwent curative surgery (29.1\%). In 16 patients (12.7\%), surgery was followed by adjuvant chemotherapy. The median overall survival of patients with ICC was 213 days.

The clinical features of hepatitis-B-associated ICC are listed in Table 2. Hepatitis B-associated ICC patients 
Table 1. Baseline characteristics of the study group

\begin{tabular}{|c|c|}
\hline Variable & Value \\
\hline Total patients & 131 \\
\hline Age, yr & $66.6 \pm 11.7$ \\
\hline \multicolumn{2}{|l|}{ Sex } \\
\hline Men & $74(56.5)$ \\
\hline Female & $57(43 \cdot 5)$ \\
\hline \multicolumn{2}{|l|}{ Hepatitis } \\
\hline $\mathrm{HBV}$ & $19(14 \cdot 5)$ \\
\hline $\mathrm{HCV}$ & $4(3.1)$ \\
\hline \multicolumn{2}{|l|}{ TNM stage } \\
\hline I & $19(14 \cdot 5)$ \\
\hline II & $13(9.9)$ \\
\hline III & $3(2.3)$ \\
\hline Iva & $45(34 \cdot 4)$ \\
\hline $\mathrm{IVb}$ & $51(38.9)$ \\
\hline Curative surgery & $37(29.1)$ \\
\hline \multicolumn{2}{|l|}{ Chemotherapy } \\
\hline Adjuvant & $16(12.7)$ \\
\hline Palliative & $62(49.2)$ \\
\hline Survival, day & $213(104.0-440.0)$ \\
\hline
\end{tabular}

Values are presented as mean $\pm \mathrm{SD}$, number (\%), or median (interquartile range).

$\mathrm{HBV}$, hepatitis virus B; HCV, hepatitis virus C; TNM, tumor-node-metastasis.

were significantly younger (6.75 [interquartile range, IQR, 60.5 to 76] vs. 57 [IQR, 52.0 to 64.0], $p=0.002$ ) with higher albumin (3.6 [IQR, 3.3 to 4.0 ] vs. 3.9 [IQR, 3.4 to $4.3], p=0.044$ ) and higher AFP (3.0 [IQR, 2.5 to 5.0] vs. 7.0 [IQR, 3.0 to 190.8], $p=0.016$ ) than those without hepatitis. There were no significant differences in other clinical indices, including bilirubin level, CA 19-9 level, or TNM stage. Hepatitis-C-associated ICC patients were not significantly different in clinical indices.

Overall survival rates of ICC patients with and without hepatitis were not significantly different $(p=0.279)$. In addition, there was no significant difference in overall survival with HBV infection status $(p=0.164)$ (Fig. 2). Table 3 describes the prognostic factors for survival identified in the study group. In univariate analyses, factors associated with survival included age $\geq 65$ years $(p=$ 0.005), albumin $\geq 3.5 \mathrm{~g} / \mathrm{dL}(p=0.025)$, CA 19-9 $\geq 100 \mathrm{ng} /$ $\mathrm{mL}(p<0.001)$, T stage $\left(\mathrm{T}_{1}-\mathrm{T}_{3}\right)$ at diagnosis $(p<0.001)$, presence of lymph node invasion $(p<0.001)$, presence

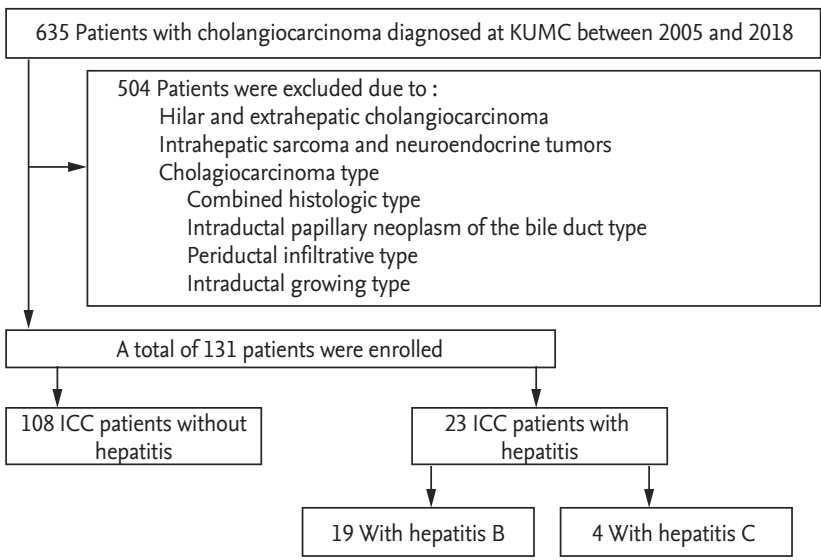

Figure 1. Flow diagram of selection and disposition of patients. KUMC, Konkuk University Medical Center; ICC, intrahepatic cholangiocarcinoma.

of metastases at diagnosis $(p<0.001)$, adjuvant chemotherapy after surgery $(p=0.004)$ and curative surgery $(p$ $<0.001)$. The median survival of ICC patients with and without hepatitis was 280 and 213 days, respectively. Survival rates did not differ significantly between the two groups $(p=0.279)$. In addition, the median survivals of patients with hepatitis $\mathrm{B}$, hepatitis $\mathrm{C}$, and without hepatitis were $302,128.5$, and 213 days, respectively. Survival rates did not differ significantly among the three groups ( $p=0.166$ vs. $p=0.603$ ). In multivariate analyses, CA 19-9 $<100 \mathrm{ng} / \mathrm{mL}(p<0.001)$, T stage $\left(\mathrm{T}_{1}-\mathrm{T}_{3}\right)$ at diagnosis $(p$ $=0.042)$, the absence of lymph node invasion $(p=0.043)$, and curative surgery $(p=0.033)$ were significant and independent predictors of a more favorable outcome.

\section{DISCUSSION}

The incidence of ICC is increasing, generating clinical interest in improving outcomes and identifying prognostic factors. Recently, many studies have indicated that HBV and HCV infection are statistically associated with ICC $[4,5,8]$. HBV and HCV infection are endemic in Korea. In our study, $17.6 \%$ of patients with ICC (23/131) were positive for HBV or HCV infection. Although we explored the prognostic value of hepatitis virus infection, we found no significant difference in overall survival between ICC patients with and without hepatitis, although some clinical features did differ between the two groups. 
Table 2. Clinical features of intrahepatic cholangiocarcinoma patients with hepatitis B virus infection and without hepatitis virus infection

\begin{tabular}{|c|c|c|c|}
\hline Variable & Without hepatitis & With hepatitis B & $p$ value \\
\hline Total patients & $108(82.4)$ & $19(14.5)$ & \\
\hline Mean age, yr & $67.5(60.5-76.0)$ & $57.0(52.0-64.0)$ & 0.002 \\
\hline \multicolumn{4}{|l|}{ Sex } \\
\hline Men & 61 & 10 & NS \\
\hline Women & 47 & 9 & \\
\hline Bilirubin, mg/dL & $0.7\left(0.5^{-1.1}\right)$ & $0.8(0.6-1.1)$ & NS \\
\hline Albumin, mg/dL & $3.6(3 \cdot 3-4 \cdot 0)$ & $3 \cdot 9(3 \cdot 4-4 \cdot 3)$ & 0.044 \\
\hline $\mathrm{CA} 19-9, \mathrm{mg} / \mathrm{dL}$ & $81.1(16.5-877.6)$ & $149 \cdot 3(19.5-756.3)$ & NS \\
\hline AFP, mg/dL & $3.0(2.5-5.0)$ & $7.0(3.0-190.8)$ & 0.016 \\
\hline CTP score & $5(5-6)$ & $5(5-5)$ & $\mathrm{NS}$ \\
\hline TNM stage & & & NS \\
\hline I & $15(13 \cdot 9)$ & $4(21.1)$ & \\
\hline II & $10(9.3)$ & $2(10.5)$ & \\
\hline III & $3(2.8)$ & o & \\
\hline IV a & $37(34 \cdot 3)$ & $6(31.6)$ & \\
\hline IV b & $43(39.8)$ & $7(36.8)$ & \\
\hline Mean survival, day & $213(105-435)$ & $302(96-686)$ & NS \\
\hline HBV viral load, IU/mL & & $231(20-409)$ & \\
\hline \multicolumn{4}{|l|}{ Antiviral treatment } \\
\hline Yes & & $8(42.1)$ & \\
\hline No & & $11(57 \cdot 9)$ & \\
\hline
\end{tabular}

Values are presented as number (\%) or median (interquartile range).

NS, no statistically significant; CA 19-9, carbohydrate antigen 19-9; AFP, $\alpha$-fetoprotein; CTP, Child-Turcotte-Pugh; TNM, tumor-node-metastasis; HBV, hepatitis B virus.

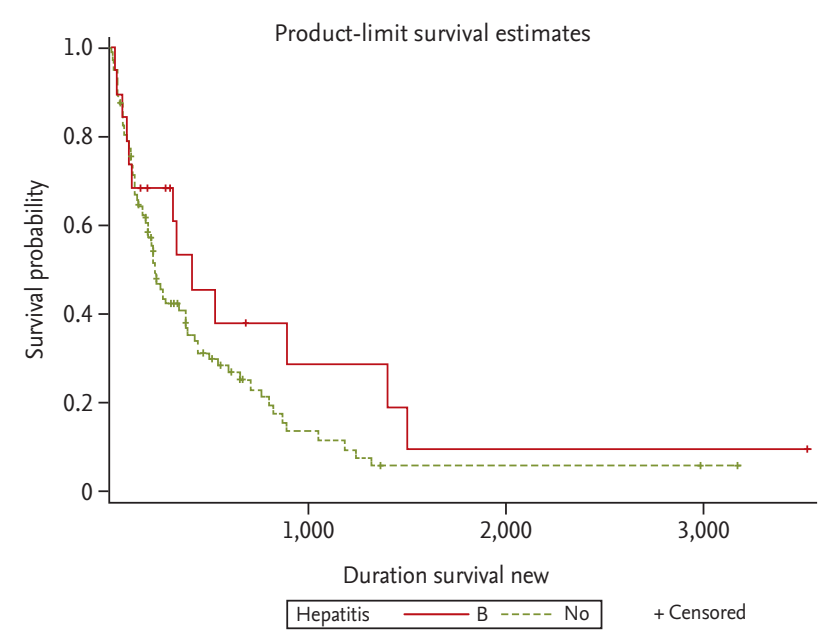

Figure 2. Kaplan-Meier curve of overall survival with hepatitis B virus (HBV) infection status. There are no significant differences in overall survival between the groups with and without HBV infection $(p=0.164)$.
Cholangiocarcinoma is more common in older patients [9]. ICC typically presents sporadically as a discrete intrahepatic mass in patients over 65 years of age [10]. In our study, the mean age of patients at the time of ICC diagnosis was 66.6 years; in patients with underlying HBV infection, ICC diagnosis occurred at an earlier age, with a mean age at presentation of 57.0 years $(p=0.002)$ (Table 2). Although these patients were diagnosed at an earlier age, most had metastatic disease or unresectable tumors at the time of diagnosis. This may be due to specific mechanisms induced by hepatitis virus infection, but this remains unclear. In several recent studies, HBV nucleic acid and HCV ribonucleic acid (RNA) have been identified in cholangiocarcinoma cells. These findings indicate that hepatitis viruses may induce cholangiocyte proliferation and dysplasia that leads to ICC development [11-14]. AFP is the most common marker for fetal 
Table 3. Univariate and multivariate analysis of factors associated with the survival of all patients with intrahepatic cholangiocarcinoma

\begin{tabular}{|c|c|c|c|c|c|}
\hline \multirow{2}{*}{ Variable } & \multirow{2}{*}{ Number } & \multirow{2}{*}{ Median survival } & \multirow{2}{*}{$\begin{array}{l}\text { Univariate analysis } \\
\qquad p \text { value }\end{array}$} & \multicolumn{2}{|c|}{ Multivariate analysis } \\
\hline & & & & $\mathrm{HR}(95 \% \mathrm{CI})$ & $p$ value \\
\hline \multicolumn{6}{|l|}{ Age, yr } \\
\hline$<65$ & 55 & 302 & & & \\
\hline$\geq 65$ & 66 & 183.5 & 0.005 & $1.415(0.856-2.339)$ & 0.176 \\
\hline \multicolumn{6}{|l|}{ Hepatitis } \\
\hline No & 98 & 213 & & & \\
\hline Hepatitis B & 19 & 302 & 0.166 & & \\
\hline Hepatitis C & 4 & 128.5 & 0.603 & & \\
\hline \multicolumn{6}{|c|}{ Hepatitis B or C } \\
\hline No & 98 & 213 & & & \\
\hline Yes & 23 & 280 & 0.279 & & \\
\hline \multicolumn{6}{|l|}{ CTP score } \\
\hline$<7$ & 100 & 216.5 & & & \\
\hline$\geq 7$ & 21 & 139 & 0.570 & & \\
\hline \multicolumn{6}{|l|}{ Albumin, g/dL } \\
\hline$<3.5$ & 40 & $119 \cdot 5$ & & & \\
\hline$\geq 3 \cdot 5$ & 81 & 268 & 0.025 & $0.875(0.522-1.467)$ & 0.612 \\
\hline \multicolumn{6}{|c|}{ Bilirubin, mg/dL } \\
\hline$<3.0$ & 111 & 217 & & & \\
\hline$\geq 3.0$ & 10 & 71 & 0.209 & & \\
\hline \multicolumn{6}{|c|}{$\mathrm{CA}$ 19-9, ng/mL } \\
\hline$<100$ & 60 & 291 & & & \\
\hline$\geq 100$ & 55 & 180 & $<0.001$ & $2.378(1.499-3.773)$ & $<0.001$ \\
\hline \multicolumn{6}{|l|}{ T stage } \\
\hline $\mathrm{T}_{1}-\mathrm{T}_{3}$ & 77 & 338 & & & \\
\hline $\mathrm{T}_{4}$ & 44 & 124 & $<0.001$ & $1.811(1.022-3.210)$ & 0.042 \\
\hline \multicolumn{6}{|l|}{ Lymph node } \\
\hline Negative & 45 & 386 & & & \\
\hline Positive & 76 & 159 & $<0.001$ & $1.756(1.018-3.027)$ & 0.043 \\
\hline \multicolumn{6}{|c|}{ Distant metastasis } \\
\hline No & 76 & 291 & & & \\
\hline Yes & 45 & 122 & $<0.001$ & $1.219(0.729-2.038)$ & 0.449 \\
\hline \multicolumn{6}{|c|}{ Adjuvant chemotherapy } \\
\hline Yes & 15 & 612 & 0.004 & $0.615(0.230-1.642)$ & 0.322 \\
\hline No & 105 & 194 & & & \\
\hline \multicolumn{6}{|c|}{ Palliative chemotherapy } \\
\hline Yes & 60 & 248.5 & 0.713 & & \\
\hline No & 60 & 194.5 & & & \\
\hline \multicolumn{6}{|c|}{ Curative surgery } \\
\hline Yes & 36 & 538 & $<0.001$ & $0.426(0.194-0.934)$ & 0.033 \\
\hline No & 84 & 144.5 & & & \\
\hline
\end{tabular}


Table 3. Continued

\begin{tabular}{|c|c|c|c|c|c|}
\hline \multirow{2}{*}{ Variable } & \multirow{2}{*}{ Number } & \multirow{2}{*}{ Median survival } & \multirow{2}{*}{$\begin{array}{l}\text { Univariate analysis } \\
\text { p value }\end{array}$} & \multicolumn{2}{|c|}{ Multivariate analysis } \\
\hline & & & & $\operatorname{HR}(95 \% \mathrm{CI})$ & $p$ value \\
\hline \multicolumn{6}{|l|}{ Tumor size, $\mathrm{cm}$} \\
\hline$<5$ & 20 & 538 & & & \\
\hline$\geq 5$ & 12 & 561.5 & 0.518 & & \\
\hline \multicolumn{6}{|l|}{ Surgical procedure } \\
\hline Major resection & 16 & 406 & 0.282 & & \\
\hline Minor resection & 16 & 612 & & & \\
\hline \multicolumn{6}{|l|}{ Resection margin } \\
\hline Negative & 26 & 406 & & & \\
\hline Positive & 7 & 612 & 0.959 & & \\
\hline \multicolumn{6}{|l|}{ Tumor differentiation } \\
\hline Well or moderate & 22 & 579.6 & & & \\
\hline Poor or un-differentiation & 10 & $1,242.3$ & 0.252 & & \\
\hline \multicolumn{6}{|l|}{ Perineural invasion } \\
\hline Yes & 4 & 962 & 0.726 & & \\
\hline No & 29 & 518 & & & \\
\hline \multicolumn{6}{|l|}{ Portal vein invasion } \\
\hline Yes & 5 & 280 & 0.379 & & \\
\hline No & 27 & 612 & & & \\
\hline \multicolumn{6}{|l|}{ Microvascular invasion } \\
\hline Yes & 13 & 377 & 0.150 & & \\
\hline No & 19 & 588 & & & \\
\hline \multicolumn{6}{|l|}{ Histologic cirrhosis } \\
\hline Yes & 4 & $744 \cdot 5$ & 0.687 & & \\
\hline No & 29 & 518 & & & \\
\hline
\end{tabular}

Median survival is expressed in days.

HR, hazard ratio; CI, confidence interval; CTP, Child-Turcotte-Pugh; CA 19-9, carbohydrate antigen 19-9.

hepatoblasts, hepatic progenitor cells (HPCs), or HCC. This study found that serum AFP levels $>20 \mathrm{ng} / \mathrm{mL}$ were present in about $30.4 \%$ of hepatitis-positive ICC patients [15]. HBV and HCV cause viral hepatitis and may cause cancer by inducing chronic inflammation in both hepatocytes and cholangiocytes, leading to high levels of serum AFP. Recently, two clinical studies implied that both hepatitis-associated ICC and HCC share a common carcinogenesis process and that both arise from HPC $[11,15]$. In this study, the incidence of AFP $>20 \mathrm{ng} / \mathrm{mL}$ was much higher in hepatitis-associated ICC than in ICC patients without hepatitis (30.4\% vs. 9.3\%). These findings may imply that, during the process of malignant trans- formation, HPCs retain their ability to produce AFP.

Based on gross morphological features, ICC can be classified into three subtypes: mass-forming, periductal-infiltrating, and intraductal. Etiologies involving distinct molecular pathways may be associated with ICC subtypes. For example, ICC associated with hepatolithiasis and Clonorchis sinensis infection nearly always has an intraductal growth pattern [16]. In our study, although six patients were excluded based on exclusion criteria, we found that hepatitis-associated ICC tended to be of the mass-forming type (23/29). As noted above, it has been suggested that hepatitis-associated ICC and HCC share a common disease mechanism. 
In our study, approximately $75 \%$ of patients had TNM stage III or IV and unresectable tumors at the time of diagnosis. Better survival was observed in patients with CA $19-9<100 \mathrm{ng} / \mathrm{mL}$, with T stage 1 to 3 at diagnosis, and without lymph node invasion. In addition, curative surgery was better for survival if curative surgical resection was possible. Tumor size, tumor differentiation, anatomical resection, perineural invasion, presence of vascular invasion, and cirrhosis, which have been reported to be associated with prognosis in previous studies, were not statistically significant in our study [17-22].

In conclusion, ICC is associated with a poor prognosis; therefore, an improved understanding of the factors that determine patient outcomes is required. In many recent studies, HBV and HCV are possible risk factors for ICC development. In this study, we did not find a prognostic value for hepatitis virus status in ICC patients; however, patients with hepatitis-associated ICC showed several clinical features that differed from those in patients without hepatitis. The limitations of this study include its small sample size and retrospective nature. Therefore, a larger prospective study is needed to clarify the prognostic role of HBV and HCV infection for ICC.

\section{KEY MESSAGE}

1. Hepatitis B virus (HBV) or hepatitis C virus (HCV) may be involved in the development of intrahepatic cholangiocarcinoma (ICC).

2. While hepatitis influenced a number of clinical features in ICC patients, it did not affect survival rate.

3. Prognostic factors influencing survival outcomes with ICC were carbohydrate antigen 19-9 level, T stage, the presence of lymph node metastasis, and curative surgery.

\section{Conflict of interest}

No potential conflict of interest relevant to this article was reported.

\section{Acknowledgments}

We would like to show our greatest appreciation to all professors of the Konkuk University Medical Center, who provided helpful comments and suggestions.

\section{REFERENCES}

1. Zhang H, Yang T, Wu M, Shen F. Intrahepatic cholangiocarcinoma: epidemiology, risk factors, diagnosis and surgical management. Cancer Lett 2016;379:198-205.

2. Lafaro KJ, Cosgrove D, Geschwind JF, Kamel I, Herman JM, Pawlik TM. Multidisciplinary care of patients with intrahepatic cholangiocarcinoma: updates in management. Gastroenterol Res Pract 2015;2015:860861.

3. Bergquist A, von Seth E. Epidemiology of cholangiocarcinoma. Best Pract Res Clin Gastroenterol 2015;29:221-232.

4. Zhou Y, Zhao Y, Li B, et al. Hepatitis viruses infection and risk of intrahepatic cholangiocarcinoma: evidence from a meta-analysis. BMC Cancer 2012;12:289

5. Matsumoto K, Onoyama T, Kawata S, et al. Hepatitis B and $\mathrm{C}$ virus infection is a risk factor for the development of cholangiocarcinoma. Intern Med 2014;53:651-654.

6. Wang Z, Sheng YY, Dong QZ, Qin LX. Hepatitis B virus and hepatitis $\mathrm{C}$ virus play different prognostic roles in intrahepatic cholangiocarcinoma: a meta-analysis. World J Gastroenterol 2016;22:3038-3051.

7. Zhou HB, Hu JY, Hu HP. Hepatitis B virus infection and intrahepatic cholangiocarcinoma. World J Gastroenterol 2014;20:5721-5729.

8. Lee TY, Lee SS, Jung SW, et al. Hepatitis B virus infection and intrahepatic cholangiocarcinoma in Korea: a case-control study. Am J Gastroenterol 2008;103:1716-1720.

9. Shimada M. Highlights of topic "Intrahepatic cholangiocarcinoma: recent advancements in pathogenesis, diagnosis and treatment". J Hepatobiliary Pancreat Sci 2015;22:91-93.

10. Dhanasekaran R, Hemming AW, Zendejas I, et al. Treatment outcomes and prognostic factors of intrahepatic cholangiocarcinoma. Oncol Rep 2013;29:1259-1267.

11. Lee CH, Chang CJ, Lin YJ, Yeh CN, Chen MF, Hsieh SY. Viral hepatitis-associated intrahepatic cholangiocarcinoma shares common disease processes with hepatocellular carcinoma. Br J Cancer 2009;100:1765-1770.

12. Perumal V, Wang J, Thuluvath $\mathrm{P}$, Choti M, Torbenson M. Hepatitis $\mathrm{C}$ and hepatitis B nucleic acids are present in intrahepatic cholangiocarcinomas from the United States. Hum Pathol 2006;37:1211-1216.

13. Chen RF, Li ZH, Zou SQ, Chen JS. Effect of hepatitis C virus core protein on modulation of cellular proliferation and apoptosis in hilar cholangiocarcinoma. Hepatobiliary Pancreat Dis Int 2005;4:71-74. 
14. Torbenson M, Yeh MM, Abraham SC. Bile duct dysplasia in the setting of chronic hepatitis $\mathrm{C}$ and alcohol cirrhosis. Am J Surg Pathol 2007;31:1410-1413.

15. Zhou H, Wang H, Zhou D, et al. Hepatitis B virus-associated intrahepatic cholangiocarcinoma and hepatocellular carcinoma may hold common disease process for carcinogenesis. Eur J Cancer 2010;46:1056-1061.

16. Wu ZF, Yang N, Li DY, Zhang HB, Yang GS. Characteristics of intrahepatic cholangiocarcinoma in patients with hepatitis B virus infection: clinicopathologic study of resected tumours. J Viral Hepat 2013;20:306-310.

17. Hanazaki K, Kajikawa S, Shimozawa N, et al. Prognostic factors of intrahepatic cholangiocarcinoma after hepatic resection: univariate and multivariate analysis. Hepatogastroenterology 2002;49:311-316.

18. Kawarada Y, Yamagiwa K, Das BC. Analysis of the relationships between clinicopathologic factors and survival time in intrahepatic cholangiocarcinoma. Am J Surg 2002;183:679-685.

19. Song GW, Lee SG, Lee YJ, et al. Analysis of survival and factors affecting the survival after surgical resection of peripheral cholangiocarcinoma: 318 cases in single institute. Korean J Hepatol 2007;13:208-221.

20. Endo I, Gonen M, Yopp AC, et al. Intrahepatic cholangiocarcinoma: rising frequency, improved survival, and determinants of outcome after resection. Ann Surg 2008;248:84-96.

21. Shirai K, Ebata T, Oda K, et al. Perineural invasion is a prognostic factor in intrahepatic cholangiocarcinoma. World J Surg 2008;32:2395-2402.

22. Yuan L, Luo X, Lu X, et al. Comparison of clinicopathological characteristics between cirrhotic and non-cirrhotic patients with intrahepatic cholangiocarcinoma: a largescale retrospective study. Mol Clin Oncol 2017;7:615-622. 